\title{
LE REFLEXE SYMPATHO-GALVANIQUE (RSG) ET LA SYMPATHECTOMIE
}

\author{
JaCQUes R. BouCher, M.D., F.R.C.P.(C), MaURICE FALARDEAU, M.D., F.R.C.S.(C), \\ Roger Plante, M.D., F.R.C.S.(C), JaCQUeS AUdet, M.D., C.R.C.P.(C), \\ et ALAin JanNard, M.D. ${ }^{*}$
}

Nous Avons tous été étonnés de certains résultats spectaculaires d'une sympathectomie mais parfois également désappointés de certains échecs inattendus. Nous avons bien recherché des moyens "médicaux" de prévoir les bons et les mauvais sujets: ${ }^{1-3}$ bloc du sympathique, anesthésie épidurale, pléthysmographie, etc. A l'école de Lewis, ${ }^{4}$ nous avons essayé le test du SGR (sympatho-galvanic reflex) que nous traduisons RsG (réflexe sympatho-galvanique). Dans cette étude, nous rapportons notre expérience de la corrélation entre le test et le résultat définitif de la sympathectomie.

Nous ne voulons pas établir lindication de la sympathectomie ni louer ses mérites ni excuser ses torts. ${ }^{5,6}$ Nous savons que la sympathectomie a été utilisée pour plusieurs raisons plus ou moins bien définies ${ }^{3}$ et a donc donné des résultats inconstants, difficiles à interpréter; aujourdhui les malades sont investigués par angiographie et nous visualisons les candidats à une chirurgie directe. Nous admettons la supériorité de cette méthode à l'approche indirecte (sympathectomie) ${ }^{7,8} \mathrm{Il}$ existe cependant plusieurs cas où la chirurgie de reconstruction est impossible: il faut alors penser à faire une sympathectomie. ${ }^{9}$

Si nous apportons une amélioration subjective et/ou objective et si nous évitons l'amputation pour une période suffisamment longue, voir définitive, nous avons aidé notre malade. La place de la sympathectomie quoique modeste dans notre esprit peut quand même être fort utile pour le malade. La mortalité étant presque nulle, il n'y a rien à perdre et peut-être un membre à sauver. ${ }^{10,11}$

\section{Phrsiologre}

L'explication physiologique de ce phénomène remonte à la fin du $19^{\text {ième }}$ siècle, alors que Féré en 1888 a constaté la présence d'une activité électrique à la peau. ${ }^{12,13}$ Cependant c'est à Tarchanoff qu'on doit la description d'un réflexe mesuré sur la peau des mains, et qui pouvait être déclenché par une variété de stimuli. ${ }^{14}$ Cest Veraguth en 1906 qui donna le terme de "réflexe psycho-galvanique." ${ }^{15}$ Ce réflexe a ensuite été utilisé pour une foule d'applications pseudoscientifiques. Enfin en 1955, Lewis ${ }^{4}$ a repris ce travail pour évaluer les résultats de blocs sympathiques lombaires et lui a donnée le nom que nous employons aujourd'hui, c'est-à-dire celui de réflexe sympatho-galvanique (RSG).

\footnotetext{
-Département de Chirurgie de l'Université de Montréal, Montréal, Québec. Dis. Boucher et Audet sont anesthésistes à l'Hôpital Notre-Dame, Montréal. Drs. Falardeau et Plante sont chirurgiens à l'Hôpital Notre-Dame, Montréal. Dr. Jannard est interne.
}

504

Canad. Anaesth. Soc. J., vol. 17, no. 5, September 1970 
La nature exacte de ce réflexe a été étudiée par plusieurs auteurs dont Schilf et Schuberth, ${ }^{18}$ Tarchanoff ${ }^{14}$ et McClendon et Hemingway. ${ }^{17}$ Ces auteurs ont démontré que les voies efférentes de ce réflexe sont sympathiques et que le réflexe peut être causé par une variété de stimuli. L'activité décelée serait due à une décharge électrique venant des cellules mêmes qui bordent les glandes sudoripares. De plus, l'activité électrique qui détermine l'enregistrement graphique de ce réflexe ne peut être décelée quaux mains et aux pieds parce que une partie des glandes sudoripares est innervée par des fibres post-ganglionnaires adrénergiques alors que les glandes sudoripares situées ailleurs dans l'organisme sont innervées par des fibres post-ganglionnaires cholinergiques. ${ }^{18}$

\section{MÉthode et Technique}

Les enregistrements de RSG que nous avons faits, l'ont été à l'aide d'un électrocardiographe de marque Sanborn, Modèle 780. Cet appareil est un moniteur de soins intensifs et sa corde ne comporte que trois électrodes dont deux actives, la troisième servant d'électrode indifférente. Cet appareil comporte donc un dispositif d'enregistrement et convient parfaitement à nos buts. Il suffit d'appliquer les deux électrodes actives au membre à examiner et d'appliquer l'électrode indifférente à l'autre membre (Figs. 1 et 2). L'enregistrement se fait à la vitesse de 25 $\mathrm{mm} / \mathrm{sec}$ et donne des formes différentes selon l'intensité de la réponse sympathique (Fig. 3). L'absence d'activité sympathique s'inscrit comme une ligne droite. L'activité sympathique positive a été divisée en: marquée, moyenne, minime, et nulle.

\section{MatérIel Clinique et Indication}

Nous avons relevé seulement les cas des malades qui ont eu un RSG de 1966 à 1969. Ceci n'est pas une étude systématique mais rétrospective. En effet la majo-

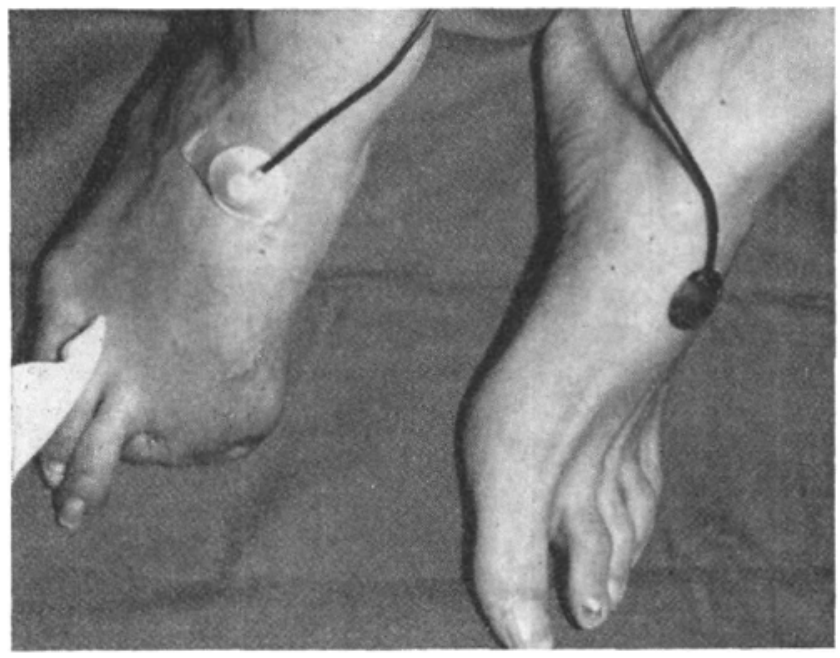

Figure 1 


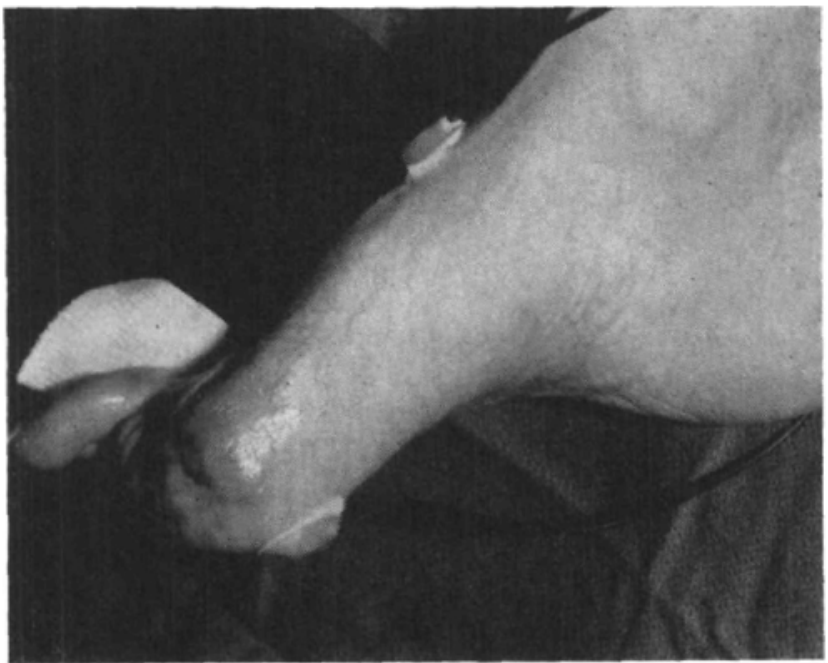

Figure 2
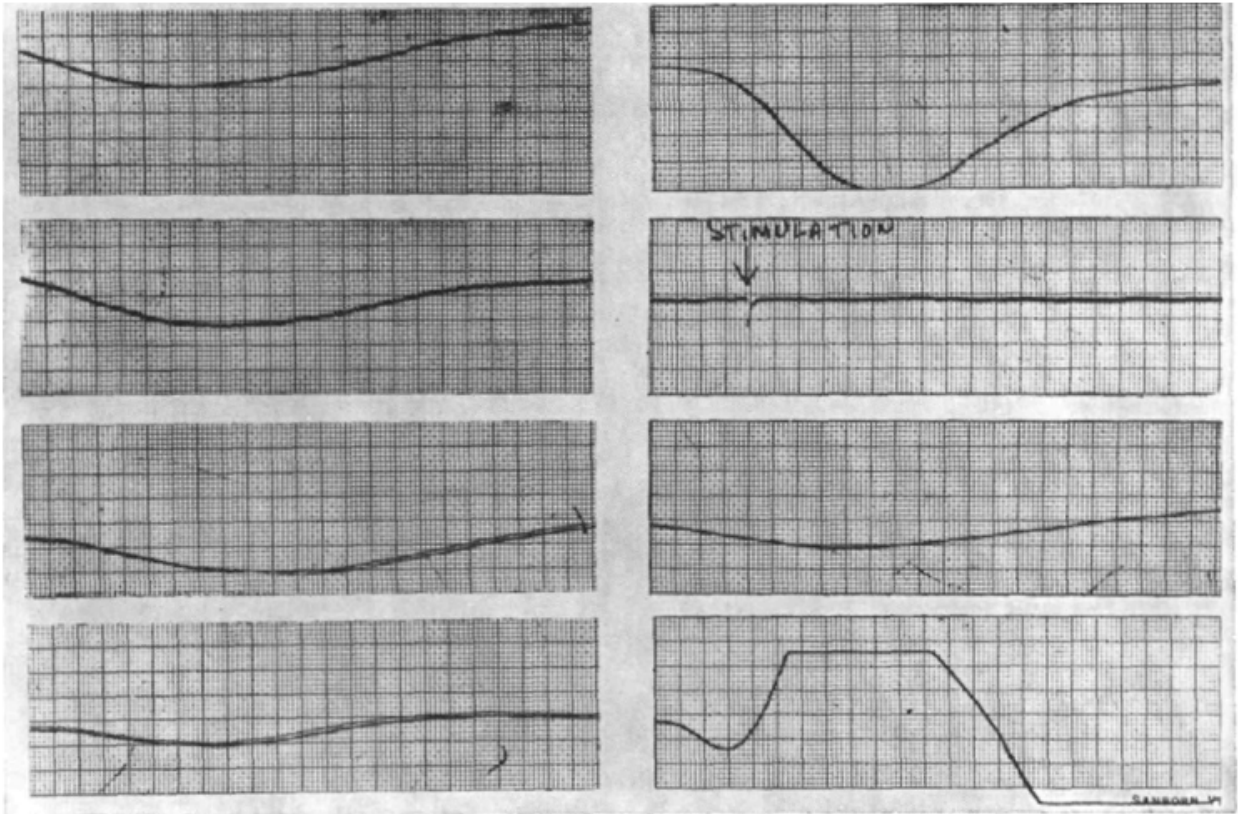

Figure 3. Tracés d'activité sympathetique.

rité de ces malades ont été évalués selon une opinion basée sur la clinique et une investigation radiographique; ces patients dont nous faisons l'étude ont été traités selon des critères cliniques. Nous faisons une corrélation entre l'évolution de ces malades et celle que nous aurait laissé prévoir le RSG.

Il faut ajouter aussi que nous n'avons pas relevé les dossiers de tous les malades qui ont eu une sympathectomie s'il n'y a pas eu de test de RSG fait à cette occa- 
sion. De plus, la majorité des malades ont été traités selon les schemes habituels sans avoir de RSG.

Nous avons donc 75 malades dont 52 hommes et 23 femmes (Tableau I). Ceci constitue l'evolution de 98 membres ( 46 droits et 52 gauches) car nous avons considéré seulement les membres qui présentaient une pathologie actuelle au moment du test RSG. Le Tableau II montre la disposition des âges et le sexe des malades. Enfin, il faut considérer que 16 de ces malades étaient diabétiques, la

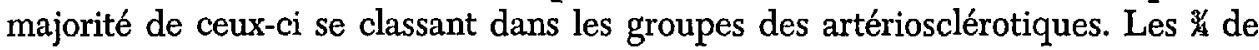
ces malades ont été traités par les deux chirurgiens et tous les RSG ont été faits par les deux anesthésistes.

Les malades faisant partie de cette étude avaient des pathologies qui, classiquement, peuvent être traitées par la sympathectomie. Nous avons considéré comme échec soit la non amélioration de la raison principale, i.e. sudation, douleur, instabilité vasculaire, soit l'amputation s'il s'agissait d'ulcération ou de névrite ischémique sévère. L’opération proposée était toujours la sympathectomie. On voit que certains membres ont été opérés (68) ou non opérés (30).

Bien que la majorité de ces malades avaient une artériosclérose oblitérante marquée avec ou sans ulcération, nous avons quand même 10 cas de maladie de Buerger, 4 cas de causalgie, 11 cas d'hyperhydrose, 14 cas de syndrome ou maladie de Raynaud.

Il existe d'autres indications de sympathectomie telles que le pied froid, ${ }^{19}$ la

\begin{tabular}{lr}
\multicolumn{2}{c}{ TABLEAU I } \\
\multicolumn{2}{c}{ ANalyse } \\
\hline Nombre de malades & $\mathbf{7 5}$ \\
Sexe & \\
hommes & 52 \\
femmes & 23 \\
Nombre de membres & 98 \\
droits & 46 \\
gauches & 52 \\
Diabete associé & 16 \\
Artériosclériose & 15 \\
Mal de Buerger & 1 \\
\hline
\end{tabular}

TABLEAU II

\begin{tabular}{|c|c|c|c|c|}
\hline Age & Numéro du patient & Hommes & Femmes & Total \\
\hline $0-9$ & & & & 0 \\
\hline 10-19 & 3036 & 0 & 2 & 2 \\
\hline $20-29$ & 81019 & 1 & 2 & 3 \\
\hline $30-39$ & $440 \quad 42 \quad 46 \quad 47 \quad 48 \quad 52 \quad 57 \quad 83$ & 4 & 5 & 9 \\
\hline $40-49$ & $\begin{array}{lllllllllllllll}1 & 5 & 6 & 11 & 12 & 19 & 31 & 35 & 37 & 39 & 45 & 66 & 72 & 77 & 91\end{array}$ & 15 & 0 & 15 \\
\hline $50-59$ & $\begin{array}{lllllllllllllll}3 & 9 & 14 & 15 & 16 & 17 & 21 & 25 & 26 & 27 & 34 & 44 & 49 & 53 & 67 \\
69 & 73 & 74 & 75 & 78 & 84\end{array}$ & 15 & 6 & 21 \\
\hline $60-69$ & $24299238 \quad 5051 \quad 62 \quad 64 \quad 70 \quad 71 \quad 76 \quad 8086$ & 9 & 4 & 13 \\
\hline $70-79$ & 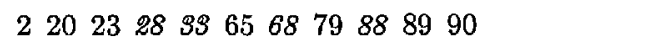 & 7 & 4 & 11 \\
\hline Plus de 80 & 43 & 1 & 0 & 1 \\
\hline Total & & 52 & 23 & 75 \\
\hline
\end{tabular}

Chiffres italiques veulent dire femme. 
maladie veineuse, la fistule artério-veineuse, l'embolie périphérique, les traumatismes, la phlébite, les ulcères trophiques, les troubles neurologiques, le lymphoedème et les engelures. Nous n'avons pas suffisamment de sujets dans ces groupes pour pouvoir en discuter les résultats.

Il faut considérer aussi que nous n'avons pas fait de sympathectomie chez les malades qui auraient bénéficié d'une angioplastie; une angioplastie est toujours une opération de choix. ${ }^{10}$

La sympathectomie a été réservée pour les cas où l'approche chirurgicale directe était impossible ou même avait déjà échoué. Cette opération n'est également pas faite de routine chez les malades qui ont eu des angioplasties et elle n'a pas été pratiquée dans le but de convertir une amputation sus-condylienne en amputation sous-condylienne.

\section{Etude de Nos Cas}

Revoyons chacune des maladies sur lesquelles portait notre étude.

\section{Hyperhydrose (Tableau III)}

Notons au départ que tous les malades, soit 11 patients atteints de cette maladie, se classaient dans le groupe RSG marqué et insistons également sur le fait que la sympathectomie les a tous améliorés de façon dramatique tel que prévu. Il est donc suggéré de pratiquer l'intervention dans ces cas mais il faudra plus de cas pour juger si réellement tous les malades ont autant d'activité sympathique et quel serait le résultat s’ils en avaient moins.

\section{Causalgie (Tableau IV)}

Quatre malades souffrant de causalgie ont été investigués au test du RSG: ceuxci ont tous des activités sympathiques mais très diverses soit un à activité marquée, deux à activité moyenne, un à activité minime. Les trois malades opérés ont confirme la prévision de l'anesthésiste c'est-à-dire qu'ils ont été des succès. Le quatrième est tout de même un malade qui avait été opéré pour causalgie (dans un autre hôpital) mais le test montrait une activité persistante: il faudrait penser à une faute de technique opératoire, ce dont nous doutons dans le cas présent, ou encore chercher à mieux comprendre le mécanisme de réadaptation physiologique après sympathectomie. ${ }^{20,21}$

\section{Maladie de Buerger (Tableau V)}

Sur dix malades qui ont eu un RSG, on constate que la répartition se fait dans les quatre groupes. Tous ceux qui ont une activité quelconque et ont été opérés ont été sauvés d'une amputation majeure sauf un cas qui a néanmoins retardé son amputation de 15 mois. C'est d'ailleurs le seul cas à activité marquée qui ait eu une sympathectomie et qui s'est soldé par un échec. Les trois malades à activité nulle qui ont eu une sympathectomie n'ont pu éviter l'amputation et rejoignent le sort de ceux qui n'ont pas eu de sympathectomie. Un seul de ces malades était par ailleurs diabétique. 


\section{Raynaud (Tableau VI)}

Treize malades sujets au syndrome ou à la maladie de Raynaud ont eu un RSG. Signalons que tous ceux-ci se classent parmi les tests à activité marquée (10) ou moyenne (3). Tous ont été opérés et tous ont été améliorés.

TABLEAU III

HYPERHYDROSE

\begin{tabular}{lrcc}
\hline Activité & Cas & Operés & Succès \\
\hline Marquée & 11 & 11 & 11 \\
Moyenne & 0 & & \\
Minimum & 0 & & \\
Nulle & 0 & & 11 \\
Total & 11 & 11 & 11 \\
\hline
\end{tabular}

TABLEAU IV

CaUsalgie

\begin{tabular}{lccccc}
\hline Activité & Cas & Operés & Non operés & Succès & Echecs \\
\hline Marquée & 1 & 1 & 0 & 1 & \\
Moyenne & 2 & 1 & 1 & 1 & 1 \\
Minimum & 1 & 1 & 0 & 1 & \\
Nulle & 0 & & & & 1 \\
Total & 4 & 3 & 1 & 3 & 1 \\
\hline
\end{tabular}

TABLEAU $V$

MALADIE DE BUERGER

\begin{tabular}{lcccc}
\hline & & \multicolumn{2}{c}{ Operés } & $\begin{array}{c}\text { Non operés } \\
\text { (échecs) }\end{array}$ \\
\cline { 3 - 5 } Activité & Cas & succès & échecs & 1 \\
\hline Marquée & 4 & 2 & 1 & \\
Moyenne & 1 & 1 & & 1 \\
Minimum & 1 & 1 & & \\
Nulle & 4 & 0 & 3 & 2 \\
Total & 10 & 4 & 4 & \\
\hline
\end{tabular}

\section{TABLEAU VI}

RAYNAUD

\begin{tabular}{lccc}
\hline \hline Activité & Cas & Operés & Succès \\
\hline Marquée & 10 & 10 & 10 \\
Moyenne & 3 & 3 & 3 \\
Minimum & 0 & & \\
Nulle & 0 & & \\
Total & 13 & 13 & 13 \\
\hline
\end{tabular}




\section{Insuffisance vasculaire périphérique sans ulcère (Tableau VII)}

Terme général, nous avons considéré ici un groupe très hétéroclite allant de la claudication plus ou moins importante, la névrite ischémique, le blocage embolique ou thrombotique plus ou moins aigü, l'obstruction d'un greffon opératoire antérieur. La répartition se fait dans tous les groupes de façon à peu près identique. Nous rapportons l'évolution de 27 membres chez 22 malades.

Il y avait 16 membres à activité marquée (6) ou moyenne (10) et tous ont connu une évolution favorable avec (11 cas) ou sans opération ( 5 cas). Sur six malades à activité minime, il y eut deux succès après opération, deux succès sans opération, et néanmoins deux échecs après l'opération. Enfin cinq cas à activité nulle n'ont pas eu de sympathectomie et il y a eu deux amputations mais quand même trois succès.

On note qu'on peut compter sur une certaine bonne évolution naturelle de la maladie. Dans la littérature, il semble admis que la claudication simple n'est pas une indication franche de sympathectomie ${ }^{22}$ et on croit en général qu'une sympathectomie après un échec de l'angioplastie n'apportera pas de résultats merveilleux.

Dans ce groupe il y avait quatre diabétiques: un malade du groupe à activité nulle a eu une sympathectomie bilatérale qui s'est quand même soldée par deux amputations; deux malades du groupe à activité moyenne ont été opérés et ont connu le succès de même que la malade du groupe à activité marquée.

TABLEAU VII

INSUFFISANCE VASCULAIRE SANS ULCÈRE (Nevrité, Claudication, Thrombose, etc.)

\begin{tabular}{lrrrrrr}
\hline & & \multicolumn{2}{c}{ Operés } & & \multicolumn{2}{c}{ Non operés } \\
\cline { 3 - 4 } \cline { 6 - 7 } Activitén & Cas & succès & échecs & & succès & échecs \\
\hline Marquée & 6 & 3 & 0 & & 3 & 0 \\
Moyenne & 10 & 8 & 0 & & 2 & 0 \\
Minimum & 6 & 2 & 2 & & 2 & 0 \\
Nulle & 5 & 0 & 0 & & 3 & 2 \\
Total & 27 & 13 & 2 & & 10 & 2 \\
\hline
\end{tabular}

Insuffisance vasculaire périphérique avec ulcère ou gangrène sèche ou non (Tableau VIII)

Ici la lésion est bien spécifique et il n'y a pas de demi-mesure; il y a une mise en demeure plus ou moins urgente: si l'ulcère ne guérit pas, il y aura amputation du membre. Nous considérons comme succès les cas où l'amputation est tout à fait locale et donc où nous jugulons le processus progressif.

Nous constatons qu'aucun de ces cas ne se classe dans la catégorie à activité marquée et leur nombre s'accroît à mesure que décroît l'activité du RsG. Nous avons pratiqué l'opération dans huit cas d'activité moyenne ce qui a donné de bons résultats dans quatre cas sur huit. Par contre sur dix cas à activité minime, six ont d'emblée été jugés non susceptibles d'être opérés et ont en effet dû être amputés: la maladie a suivi son cours inexorable. Quatre cas sélectionnés ont été 
opérés et il y a eu deux succès et deux échecs. Mais enfin 15 cas avaient des activités nulles et nous avons tenté l'opération dans six cas: il y a eu cinq échecs et un succès ce qui serait peut-être un cas de faux négatif.

En résumé, s'il y a ulcère, il existe certaines chances de succès ${ }^{23}$ lorsqu'il y a une activité et les chances sont presque nulles s'il n'y a pas d'activité au RSG.

\section{Analyses Globales (Tableau IX)}

A. Sur 32 membres qui présentaient des RSG marqués, 28 sympathectomies ont été pratiquées avec un résultat satisfaisant dans 27 cas. Le seul échec était un cas de maladie de Buerger d'évolution rapide et fatale. Les quatre lésions non opérées ont quand même vu leurs problèmes s'améliorer sauf un cas qui est celui du membre opposé de ce même Buerger qui avait constitué un échec malgré l'opération.

B. Vingt-quatre membres présentaient des RSG d'intensité moyenne et 21 ont eu une sympathectomie efficace dans 16 cas. Sur les cinq échecs, quatre patients avaient déjà des ulcérations que la sympathectomie n'a pas améliorées. Le cinqième malade présentait une névrite ischémique rebelle. Deux des trois membres non opérés ont été améliorés: il faut noter qu'il s'agissait d'insuffisance vasculaire artériosclérotique. Le cas non opéré qui s'est soldé par un échec était encore un cas de Buerger.

c. Il y avait 18 membres dont la réponse du RSG montrait une réponse minime. Nous avons pratiqué dix sympathectomies avec succès dans six cas mais échec dans quatre autres cas, dont deux cas d'ulcère et deux cas d'insuffisance vascu-

TABLEAU VIII

InSUfFisAnce VASCULAIRE AVEc UlCÈre

\begin{tabular}{|c|c|c|c|c|c|}
\hline \multirow[b]{2}{*}{ Activité } & \multirow[b]{2}{*}{ Cas } & \multicolumn{2}{|c|}{ Operés } & \multicolumn{2}{|c|}{ Non operés } \\
\hline & & succès & échecs & succès & échecs \\
\hline $\begin{array}{l}\text { Marquée } \\
\text { Moyenne } \\
\text { Minimum } \\
\text { Nulle }\end{array}$ & $\begin{array}{r}0 \\
8 \\
10 \\
15\end{array}$ & $\begin{array}{l}4 \\
2 \\
1\end{array}$ & $\begin{array}{l}4 \\
2 \\
5\end{array}$ & $\begin{array}{l}0 \\
0 \\
0\end{array}$ & $\begin{array}{l}0 \\
6 \\
9\end{array}$ \\
\hline Total & 33 & 7 & 11 & 0 & 15 \\
\hline
\end{tabular}

TABLEAU IX

RESUltat DU RSG

\begin{tabular}{lcccc}
\hline \hline Activité & $\begin{array}{c}\text { Nombre } \\
\text { total }\end{array}$ & $\begin{array}{c}\text { Nombre } \\
\text { operés }\end{array}$ & Succès & Echecs \\
\hline Marquée & 32 & 28 & 27 & $\mathbf{1}$ \\
Moyenne & 24 & 21 & 16 & 5 \\
Minimum & 18 & 10 & 6 & 4 \\
Sub-total & 74 & 59 & 49 & 10 \\
Nulle & 24 & 9 & 1 & 8 \\
Total & 98 & 68 & 50 & 18 \\
\hline
\end{tabular}


laire. Huit malades n'ont pas été opérés dont deux cas ont évolué de façon heureuse et il s'agissait de troubles artériosclérotiques.

D. Le RSG s'est avéré negatif dans 24 cas. Sur neuf cas opérés, il y eut huit échecs et le seul succès est d'autant plus remarquable qu'il s'agissait d'un malade avec ulcération. S'agirait-il d'un faux négatif? Les 15 autres cas non opérés se sont soldés par des échecs sauf encore trois cas de malades qui accusaient de la claudication sévère.

On voit donc de façon générale que plus l'activité est marquée, meilleures sont les chances de succès avec la sympathectomie et que c'est un guide fiable de succès dans 27 des 28 cas opérés dans le groupe à activité marquée ou d'échec dans huit des neuf cas opérés dans le groupe à activité nulle. D'ailleurs la réponse reste fiable dans les groupes $\mathrm{B}$ et $\mathrm{C}$ si on exclut les cas d'ulcère. Ceci constitue une expérience limitée mais il y a quelques autres aspects intéressants à souligner quant à l'activité prévue et la corrélation du test.

Il saute aux yeux que tous les cas d'hyperhydrose ont un RSG marqué et ont connu des succès dans tous les cas. Les quatre cas de causalgie avaient des activités variées et la sympathectomie les a améliorés quand même. ${ }^{24}$ Tous les cas de Raynaud avaient des activités marquées ou moyennes et ont tous été améliorés par la sympathectomie.

Cependant l'activité des cas de Buerger et d'insuffisance vasculaire ne peut être prévue. Il est malheureux de constater que la majorité des cas d'ulcère se classe parmi ceux qui ont des RSG moins actifs ou franchement négatifs et on peut donc espérer peu de bénéfices d'une sympathectomie.

Il y avait 16 malades franchement diabétiques sur 75 malades, constituant un pourcentage élevé par rapport à l'ensemble de la population et ceci montre bien l'importance de cette maladie dans l'étiologie des troubles vasculaires et l'importance de rechercher le diabète chez ces malades porteurs d'ulcère ou de troubles d'insuffisance vasculaire. Un seul de ces malades était egalement un cas de Buerger et les 15 autres malades faisaient partie du groupe artériosclérotique.

Il n'est par ailleurs pas absolu de dire (Tableau X) que tous les diabétiques se sont déjà fait une sympathectomie physiologique. ${ }^{25} \mathrm{Si} 11$ membres d'entre eux avaient un RSG négatif, cinq avaient quand même une activité minime, quatre une activité moyenne et un une activité marquée. Notons aussitôt que quatres des cinq

TABLEAU $X$

Diabitte et Artériosclérose*

\begin{tabular}{|c|c|c|c|c|c|}
\hline \multirow[b]{2}{*}{ Activité } & \multirow[b]{2}{*}{ Cas } & \multicolumn{2}{|c|}{ Operés } & \multicolumn{2}{|c|}{ Non operés } \\
\hline & & succès & échecs & succès & échecs \\
\hline $\begin{array}{l}\text { Marquée } \\
\text { Moyenne } \\
\text { Minimum } \\
\text { Nulle }\end{array}$ & $\begin{array}{r}1 \\
4 \\
5 \\
11\end{array}$ & $\begin{array}{l}1 \\
3 \\
1 \\
0\end{array}$ & $\begin{array}{l}0 \\
1 \\
2 \\
5\end{array}$ & $\begin{array}{l}0 \\
0 \\
0 \\
0\end{array}$ & $\begin{array}{l}0 \\
0 \\
2 \\
6\end{array}$ \\
\hline \multicolumn{6}{|c|}{ DIABÈTE ET MALADIE DE BUERGER } \\
\hline Nulle & 2 & 0 & 2 & 0 & 0 \\
\hline
\end{tabular}


membres classés dans le groupe artériosclérotiques à activité marquée ou moyenne ont été améliorés par la sympathectomie et que l'autre qui s'est soldé par un échec était encore un cas d'ulcération; il semble que l'activité sympathique serait encore un guide valable dans les cas de diabète puisque par ailleurs sept des huit cas à activité minime ou nulle qui ont été opérés ont connu des échecs. Le seul cas de diabète qui ne s'est pas soldé par un échec présentait une ulcération au membre supérieur après thrombose de l'artère sous-clavière. Par ailleurs les huit cas à activité minime ou nulle non opérés ont été des échecs.

Donc il est presque juste de dire qu'un ulcère chez un diabétique finira par une amputation si son RSG est nul ou minime. Cependant s'il y a une lésion qui permet une attaque chirurgicale directe de revascularisation (angioplastie), le pronostic est de beaucoup meilleur.

Il faut considérer que nous avons fait cette étude basée sur la clinique et non sur le RSG. Les malades avaient des traitements habituels. Beaucoup de malades n'ont pas eu de RSG; plusieurs malades ont eu une attaque chirurgicale directe (endartérectomie, prothèse de Dacron, greffe veineuse).

Quelle est l'importance de la vascularisation sur l'interprétation de ce test: un malade sans circulation et RSG négatif, verra-t-il son RSG revenir positif après angioplastie?

De plus, avec le temps, il faudra faire des tests d'activité avant et après les opérations et contrôler de nouveau, ce qui advient lorsque le greffon se thrombose. Cette réponse obtenue est-elle encore valable?: si la réponse est négative, est-ce parce qu'il n'y a plus de flot ou est-ce que la réponse à l’afflux sanguin a été maximale et que le malade ne sait plus répondre; si par ailleurs l'activité du RSG persiste, est-ce que le malade a encore une chance d'avoir une amélioration par la sympathectomie?21 Par ailleurs, une réponse positive nous permettrait peut être de juger de l'avantage de faire une sympathectomie en même temps que l'angioplastie. ${ }^{28}$

Nous avons quand même dans notre série sept malades qui ont déjà eu des greffes fémoro-poplitées et qui ont subi par la suite une sympathectomie pour troubles vasculaires. Une hypoxie tissulaire prolongée amène-t-elle une dégénérescence de la fibre sympathique et cette dégénérescence est-elle réversible après réinstauration d'une perfusion adéquate? Il existe donc plusieurs problèmes encore à envisager.

\section{CONCLUSION}

Nous avons étudié le réflexe sympatho-galvanique (RSG) chez 75 malades. L'activité sympathique était divisée comme suit: marquée, moyenne, minime et nulle. Les pathologies en cause étaient les suivantes: hyperhydrose, causalgie, maladie de Buerger, Raynaud, insuffisance vasculaire périphérique avec ou sans ulcère.

Les résultats nous ont montré que de façon générale, plus l'activité est marquée, meilleures sont les chances de succès après la sympathectomie et que c'est un guide fiable de succès dans 27 des 28 cas opérés dans le groupe à activité marquée ou d'échec dans huit des neuf cas opérés dans le groupe à activité nulle. 


\section{SUMMary}

We have reconsidered the psycho-galvanic reflex as described by Féré and Tarchanoff at the turn of the century and modernized by Lewis in 1955 under the name of sympatho-galvanic reflex (SGR). We have reviewed the cases of 75 patients who have had an SGR for different vascular troubles of the extremities, trying to correlate the presence of sympathetic activity with the results of a surgical sympathectomy.

The diseases involved in this survey were hyperhydrosis, causalgia, Raynaud's disease, Buerger's disease, and peripheral vascular insufficiency with or without ulceration. Our results showed that the more marked the sympathetic activity, the better the chances of surgical success. This has proven to be a reliable guide to success in 27 of 28 patients operated on with marked sympathetic activity, or to failure in eight of nine patients operated on with no sympathetic activity.

\section{BIBLIOGRAPHIE}

1. Ghtrighelli, C.; Lombardi, C.; \& Montorsi, W. Importance semeiologique de la résistance électrique cutanée comme indication à une réintervention chirurgicale sur le système sympathique lombaire. Lyon chir. 56: (1960).

2. MYERS, K. A. et al. Prediction of the Response to Lumbar Sympathectomy and arterial Reconstruction Operations. J. Cardio. Surg. 8: 327 (1967).

3. Standness, D. E. \& Beil, S. W. Peripheral Vascular Disease. Suppl., Ann. Surg. 161: 1 (1968).

4. LEwrs, L. W. Evaluation of Sympathetic Activity Following Chemical or Surgical Sympathectomy. Curr. Res. Anesth. 34: 334 (1955).

5. MEDEIRos, A. DE. The Role of Lumbar Sympathectomy in the Era of Direct Arterial Surgery. Vasc. Dis. 4: 353 (1967).

6. Montorsi, W.; Ghirughelit, C.; Traerio, G.; \& Lovorato, F. La Sympathectomie lombaire: peut-elle influer favorablement sur la claudication intermittente et sur les troubles trophiques dans les artériopathies des membres inférieurs. Angéiologie. 10: 5 (1958).

7. Kinmonth, John B.; Rob, Charles C.; \& Simeone, Fiorindo A. Vascular Surgery, London: Edward Amold (1962).

8. SZILAGYI, D. F. et al. Lumbar Sympathectomy. Current Role in the Treatment of Arteriosclerotic Occlusive Disease. Arch. Surg. 95: 753 (1967).

9. CARTIER, G. E. Réflexions sur notre attitude actuelle à l'égard des troubles circulatoires et en particulier de l'artériosclérose. Un. Médicale du Canada. Tome 94 (1965), pp. $1422-25$.

10. Bơd, A. M. The Role of Sympathectomy in the Management of Intermittent Claudication. Vasc. Dis. 3: 137 (1966).

11. Eckman, W. G., Jr. Lumbar Sympathectomy in the Treatment of Peripheral Vascular Insufficiency: A Review of 105 Consecutive Cases. Ohio Med. J. 51: 1190 (1955).

12. FÉRÉ, C. Notre sur des modifications de la tension electrique dans le corps humain. Compt. Rend. Soc. Biol. 5: 28 (1888).

13. FÉné, C. Notre sur des modifications de la résistance electrique sous l'influence des excitations sensorielles et des emotions. Compt. Rend. Soc. Biol. 5: 217 (1888).

14. TarchanofF, J. Uber die galvanischen Erscheinungen in der Haut des Menschen bei Reigungen der Sinnersorgane und bei verschieden Formen der psychischen Thätigkeit. Arch. ges. Physiol. 46: 46 (1890).

15. Veraguth, O. Das psychogalvanische Reflex-Phanomen. Monatschr. f. Psychiat. u. Neurol. 21: 387 (1907).

16. Schimf, E. \& SchUberth, A. Uber das sog. psycholgalvanische Reflexphanomen beim Frosch und seine Beziehung zum vegetativen Nervensystem. Arch. ges. Physiol. 195: 75 (1922).

17. McClendon, J. F. \& Hemingway, A. The Psychogalvani Reflex as Related to the Polarization-Capacity of the Skin. Am. J. Physiol. 94: 77 (1930). 
18. Guyton, A. C. Medical Physiology. Textbook 7, 3rd ed. Philadelphia: Saunders (1966).

19. Enjalbert, A. et al. Indications and Results of Lumbar Sympathectomy. J. Cardiovasc. Surg. 9: 146 (1968).

20. Keatinge, W. R. J. Electrical and Mechanical Responses of Arteries to Stimulation of Sympathetic Nerves. Physiol. 185: 701 (1966).

21. Parks, V. J.; Skinner, S. L.; \& Whelan, R. F. Mechanisms in the Return of Vascular Tone Following Sympathectomy in Man. Circul. Res. 9: 1026 (1961).

22. Myers, K. A. \& Invine, W. T. An Objective Study of Lumbar Sympathectomy: I. Intermittent Claudication, Brit. Med. J. 5492: 879 (1966).

23. Myers, K. A. \& Irvine, W. T. An Objective Study of Lumbar Sympathectomy: II. Skin Ischemia. Brit. Med. J. 5493: 947 (1966).

24. Martineau, J. P. \& Telmosse, F. La Sympathectomie dorsale haute par thoracotomie axillaire. Union Médicale du Canada. Tome 96 (1967), 1508-17.

25. Moorhouse, J. A. et al. Vascular Responses in Diabetic Peripheral Neuropathy. Brit. Med. J. 5492: 883 (1966).

26. Weismann, R. E. \& Upson, J. F. Use of Lumbar Sympathectomy as an adjunct to Reconstructive Arterial Surgery. Ann. Surg, 154: 788 ( 1961 ).

27. McAllister, F. F. The Place of Lumbar Sympathectomy in the Light of Recent Advances in Vascular Surgery. Bull. NY Acad. Med. 37: 433 (1961). 